\title{
Transformation of Polarized Light Information in the Central Complex of the Locust
}

\author{
Stanley Heinze, Sascha Gotthardt, and Uwe Homberg \\ Fachbereich Biologie, Tierphysiologie, Philipps-Universität Marburg, D-35032 Marburg, Germany
}

\begin{abstract}
Many insects perceive the $E$-vector orientation of polarized skylight and use it for compass navigation. In locusts, polarized light is detected by photoreceptors of the dorsal rim area of the eye. Polarized light signals from both eyes are integrated in the central complex (CC), a group of neuropils in the center of the brain. Thirteen types of CC neuron are sensitive to dorsally presented, polarized light (POL-neurons). These neurons interconnect the subdivisions of the CC, particularly the protocerebral bridge (PB), the upper and lower divisions of the central body (CBU, CBL), and the adjacent lateral accessory lobes (LALs). All POL-neurons show polarization-opponency, i.e., receive excitatory and inhibitory input at orthogonal $E$-vector orientations. To provide physiological evidence for the direction of information flow through the polarization vision network in the $\mathrm{CC}$, we analyzed the functional properties of the different cell types through intracellular recordings. Tangential neurons of the CBL showed highest signal-to-noise ratio, received either ipsilateral polarized-light input only or, together with CL1 columnar neurons, had eccentric receptive fields. Bilateral polarized-light inputs with zenith-centered receptive fields were found in tangential neurons of the PB and in columnar neurons projecting to the LALs. Together with other physiological parameters, these data suggest a flow of information from the CBL (input) to the PB and from here to the LALs (output). This scheme is supported by anatomical data and suggests transformation of purely sensory $E$-vector coding at the CC input stage to position-invariant coding of $360^{\circ}$-compass directions at the output stage.
\end{abstract}

\section{Introduction}

The central complex (CC) is a prominent group of neuropils in the center of the insect brain. It consists of the protocerebral bridge $(\mathrm{PB})$, the upper and lower divisions of the central body (CBU, $\mathrm{CBL}$ ), and the paired noduli. In all insect species studied, the $\mathrm{PB}$, the $\mathrm{CBL}$ and the $\mathrm{CBU}$ consist of linear arrays of 16 vertical columns and the CBU and CBL, in addition, of several horizontal layers (Williams, 1975; Homberg, 1991; Müller et al., 1997; Heinze and Homberg, 2008). The major building blocks of this almost crystalline structure are columnar and tangential neurons.

Evidence on the functional role of the CC is largely provided along three lines. Analysis of structural mutants in the fruit fly Drosophila melanogaster promotes a role of the CC in locomotion and leg coordination (Strausfeld, 1999; Strauss, 2002a). Behavioral learning paradigms in D. melanogaster, furthermore, suggest that the CC is essential for the memory of specific, behaviorally relevant visual features (Liu et al., 2006; Neuser et al., 2008). Electrophysiological data from locusts and crickets finally show that CC-neurons respond to the orientation of the electric field

Received April 20, 2009; revised July 16, 2009; accepted Aug. 7, 2009.

This work was supported by Deutsche Forschungsgemeinschaft Grant H0 950/16-2. We are grateful to Dr. Keram Pfeiffer for providing Spike2-scripts and for contributing to physiological data on CL1, TB1, and CP2 cells. We thank Stephan Gebhardt for contributing physiological data on TL2, CL1, and CP1 neurons, Basil el Jundi for contributing physiological data on a TL2 neuron, Ulrike Träger for providing reconstructions of TL2/3 neurons, Sebastian Richter and Manfred Peil for constructing the stimulation devices and control equipment, and Karl Heinz Herklotz for raising desert locusts.

Correspondence should be addressed to Uwe Homberg, Fachbereich Biologie, Tierphysiologie, Universität Marburg, D-35032 Marburg, Germany. E-mail: homberg@staff.uni-marburg.de.

DOI:10.1523/JNEUROSCI.1870-09.2009

Copyright $\odot 2009$ Society for Neuroscience 0270-6474/09/2911783-11\$15.00/0 vector (E-vector) of linearly polarized light (Vitzthum et al., 2002; Heinze and Homberg, 2007; Sakura et al., 2008; Heinze and Homberg, 2009). As the pattern of E-vectors in the blue sky is directly correlated with the sun's position, sensitivity to polarized light points to a role of the CC in sky compass navigation (Homberg, 2004). Specifically, the topographic representation of zenithal $E$-vectors underlying the columns of the $\mathrm{PB}$ is suited to code for global head orientation with respect to the solar azimuth (Heinze and Homberg, 2007). All lines of evidence together suggest a likely role of the insect $\mathrm{CC}$ as an internal compass required for planning of goal directed behavior.

In the CC of the desert locust, at least 13 types of neuron are part of the polarization vision network (Vitzthum et al., 2002; Heinze and Homberg, 2007; Heinze and Homberg, 2009). Three additional types of neuron, termed conditional POL-neurons, show polarization sensitivity in 50\% of recordings and appear to be recruited to the network dependent on the behavioral context (Heinze and Homberg, 2009). To analyze the direction of information flow through the polarization vision network in the CC, we compared the functional properties of the different cell types based on intracellular recordings. Quantitative comparison of background firing properties, response amplitudes and variability, tuning precision, ocular dominance, and visual field structure resulted in coherent differences between the different cell types and suggest a scheme of information flow from TL2/3 input neurons, via CL1 cells to TB1/2 neurons and, finally, to CPU1 and CP1/2 output cells. The data support a scheme of information flow within the locust CC based on morphological evidence and distribution of preferred $E$-vector orientations ( $\Phi_{\max }$ values) of the involved neurons (Heinze and Homberg, 2009). 


\section{Materials and Methods}

Animals and preparation. Adult desert locusts (Schistocerca gregaria) of both sexes were obtained from a crowded colony at the University of Marburg. Animals were raised in a 12/12 light/day photoperiod at constant temperature $\left(28^{\circ} \mathrm{C}\right)$. Preparation for electrophysiology was performed as described by Heinze and Homberg (2009). In short, animals were cold anesthetized, legs and wings were removed, and after fixing the locust to a metal holder in an upright position, the head capsule was opened. The brain was exposed and the gut, as well as the neural sheath near the recording area, was removed. The brain was kept submerged in locust saline (Clements and May, 1974) at all times to prevent desiccation.

Electrophysiology. Intracellular recordings were performed with sharp microelectrodes (resistance: 50-150 M $\Omega$ ), drawn from borosilicate capillaries ( $0.75 \mathrm{~mm}$ inner diameter, $1.5 \mathrm{~mm}$ outer diameter; Hilgenberg) using a Flaming/Brown horizontal puller (P-97, Sutter). Electrode tips were filled with $4 \%$ Neurobiotin (Vector Laboratories) in $1 \mathrm{~m} \mathrm{KCl}$. The electrode shaft was backed up with $1 \mathrm{M} \mathrm{KCl}$. Intracellular signals were amplified $(10 \times)$ with a custom made amplifier or a SEC5-LX amplifier (NPI). After sampling at a rate of at least $5 \mathrm{kHz}$ (CED1401 micro, Cambridge Electronic Design; or Digidata 1322A, Molecular Devices), signals were stored on a PC using Spike2 (Cambridge Electronic Design) or PClamp9 software (Molecular Devices). Digital high pass filtering was applied when necessary to compensate for drifting baseline. Depolarizing current was applied (1-3 nA, 1-5 min) to iontophoretically inject Neurobiotin immediately after recording.

Stimuli. Visual stimuli were produced with a xenon-arc (XBO $150 \mathrm{~W})$, passed through light guides (Schölly), to be finally presented to the animal from dorsal direction (irradiance at the animal's head: $94 \mu \mathrm{W} / \mathrm{cm}^{2}$; angular size: $2.7^{\circ}$ ). A polarizer (HN38S, Polaroid) was inserted between the end of the light guide and the animal and could be rotated in either direction by $360^{\circ}$ at velocities between 20 and $30^{\circ} \%$ s. Rotation velocity and direction were controlled via custom designed hard- and software. The light guide and polarizer were mounted on a perimeter and could thus be moved horizontally around the vertically mounted animal. Therefore, different elevations of stimuli could be tested. With few exceptions, the polarizer was rotated by $360^{\circ}$ at least once in each direction (clockwise and counter clockwise) at all tested stimulus positions. To allow for monocular stimulations, a small piece of black cardboard was used to block the light path to one eye during dorsal stimulations. The piece was placed very close to the animal, either hand held or, in most cases, positioned with a micromanipulator. Stimulus presentation was always started with dorsal, binocular stimuli, followed either by stimulation from different elevations or by monocular stimulations. At the end, a binocular, dorsal control stimulus was applied in most cases.

Histology. After Neurobiotin injection, brains were dissected out of the head capsule and fixed in a mixture of $4 \%$ paraformaldehyde, $0.25 \%$ glutaraldehyde, and $2 \%$ saturated picric acid (in $0.1 \mathrm{~m}$ phosphate buffer) overnight at $4^{\circ} \mathrm{C}$. After rinsing with $0.1 \mathrm{M}$ phosphate buffered saline (PBS), brains were incubated with Cy3-conjugated Streptavidin (Dianova, 1:1000, in PBS with $0.5 \%$ Triton X-100). Preparations were subsequently rinsed, dehydrated in an increasing ethanol series, cleared in methylsalicylate, and mounted in Permount (Fisher Scientific), as described in detail in Heinze and Homberg (2008).

Labeled neurons were scanned with a confocal microscope (Leica TCS-SP2, objective: HC PL APO 10×/0.4 Imm Corr CS; Leica Microsystems) to confirm the neuron type. For detailed analysis and reconstructions, selected preparations were rehydrated, sectioned, and stained by immunofluorescence with primary antibodies against serotonin and synapsin (Heinze and Homberg, 2008). For camera lucida reconstructions, rehydrated preparations were sectioned and incubated with peroxidaseconjugated Streptavidin (1:200; Amersham Buchler). Staining was obtained by peroxidase-mediated precipitation of $3,3^{\prime}$-diaminobenzidine tetrahydrochloride $(0.3 \mathrm{mg} / \mathrm{ml})$, intensified with nickel ammonium sulfate (Heinze and Homberg, 2008). Neuropil reconstructions are based on anti-synapsin immunolabeling and were performed with Amira 4.2 software (Visage Imaging).
General statistical procedures. Statistical comparison of quantitative data obtained from recorded POL-neurons (tuning widths, background firing rates, response amplitudes, etc.), was performed either pairwise (e.g., for comparison of ipsilateral versus contralateral stimulations) or across all cell types. Except for linear regression, SPSS software (version 11.5) was used to perform statistical tests. For all analyses, data were tested for normality with the Shapiro-Wilk test (significance level, 0.05) and for homogeneity of variance with the Levene test (significance level, 0.05). Pairwise testing was done with the Mann-Whitney $U$ test for data not distributed normally, whereas Student's $t$ test was applied for normally distributed data. One-way ANOVA (with F-statistics, combined with Tukey-honestly significant difference (HSD) post hoc analysis for multiple testing) was used to test for differences among multiple groups of data if data were normally distributed and variance was homogeneous. If this was not the case, ANOVA with Brown-Forsythe statistics, combined with Games-Howell post hoc analysis for multiple testing was applied. Significance levels of 0.05 were used in all cases. Linear regression and correlation analysis was performed in Origin6.0 software (Microcal). The correlation coefficient $\left(R_{\text {cor }}\right)$ was calculated, and the significance of the regression was tested with Student's $t$ test against a regression line with slope of zero (significance level, 0.05). Analysis of covariance (univariant ANCOVA, using SPSS) was used to compare the slopes of regression lines. Hereby, the interaction between the covariate (background frequency) and the fixed factor (cell type) was tested for significant influence on the independent variable (response strength; significance level: 0.05). This was done either including all considered groups (cell types) or only including the two groups, which were compared at the time.

SDs were used in graphic displays whenever the actual variability of the data was compared, whereas SEs were used as a measure of the precision of the mean.

Analysis of general response characteristics. Sampled spike trains were stored on PC and evaluated using Spike2-software with implemented, custom designed scripts. Action potentials were detected with threshold based event detection. The detected events were visualized as mean frequency using a gliding average algorithm at a bin size of $1 \mathrm{~s}$. Background frequency was estimated by moving a horizontal curser to the desired level in the mean frequency channel and reading out the current values of the curser position.

To calculate the strength of a polarized-light response [response amplitude $(R)$ ], each filter rotation was divided into $20^{\circ}$ bins. The mean spiking frequency over all bins was calculated, as well as the frequency within each bin. The summed, absolute difference between the mean frequency and the individual frequencies was defined as the $R$ value of that polarized-light response and reflected the magnitude of frequency modulation during the rotation. In some cases $180^{\circ}$ rotations of the polarizer were applied instead of $360^{\circ}$ rotations. Then, the resulting $R$ value was doubled to account for the different number of bins. Due to the usage of frequencies (impulses per second) rather than spike counts per bin (Labhart, 1996; Pfeiffer, 2006), $R$ values derived from rotations at different rotation velocities were directly comparable.

Background variability was calculated as the $R$ value of a spike train, when no stimulus was presented. To reveal the magnitude of spontaneous frequency modulations relative to the amplitude of polarized-light responses, we normalized the absolute background variability to the mean response amplitude of each neuron. Variability of $R$ was calculated by averaging $R$ values of all rotations from each neuron and calculating the SD thereof. These values were also normalized to the mean $R$ value of each neuron.

$\Phi_{\max }$ values were calculated with circular statistics (Rayleigh test for axial data, significance level: 0.05) as described previously (Pfeiffer et al., 2005; Heinze and Homberg, 2007; Pfeiffer and Homberg, 2007).

Evaluation of tuning curves. E-vector tuning widths were calculated using custom designed scripts in MatLab (MathWorks). Spike frequencies during $E$-vector rotation were calculated in $5^{\circ}$-wide bins. The resulting data were low-pass filtered, and the half maximal activation level was calculated. The width of the $E$-vector response at this level was defined as the tuning width. Mean tuning widths were calculated for each neuron.

For shape averaging of tuning curves, we selected five neurons of each category of POL-neuron, which showed the strongest response. This 

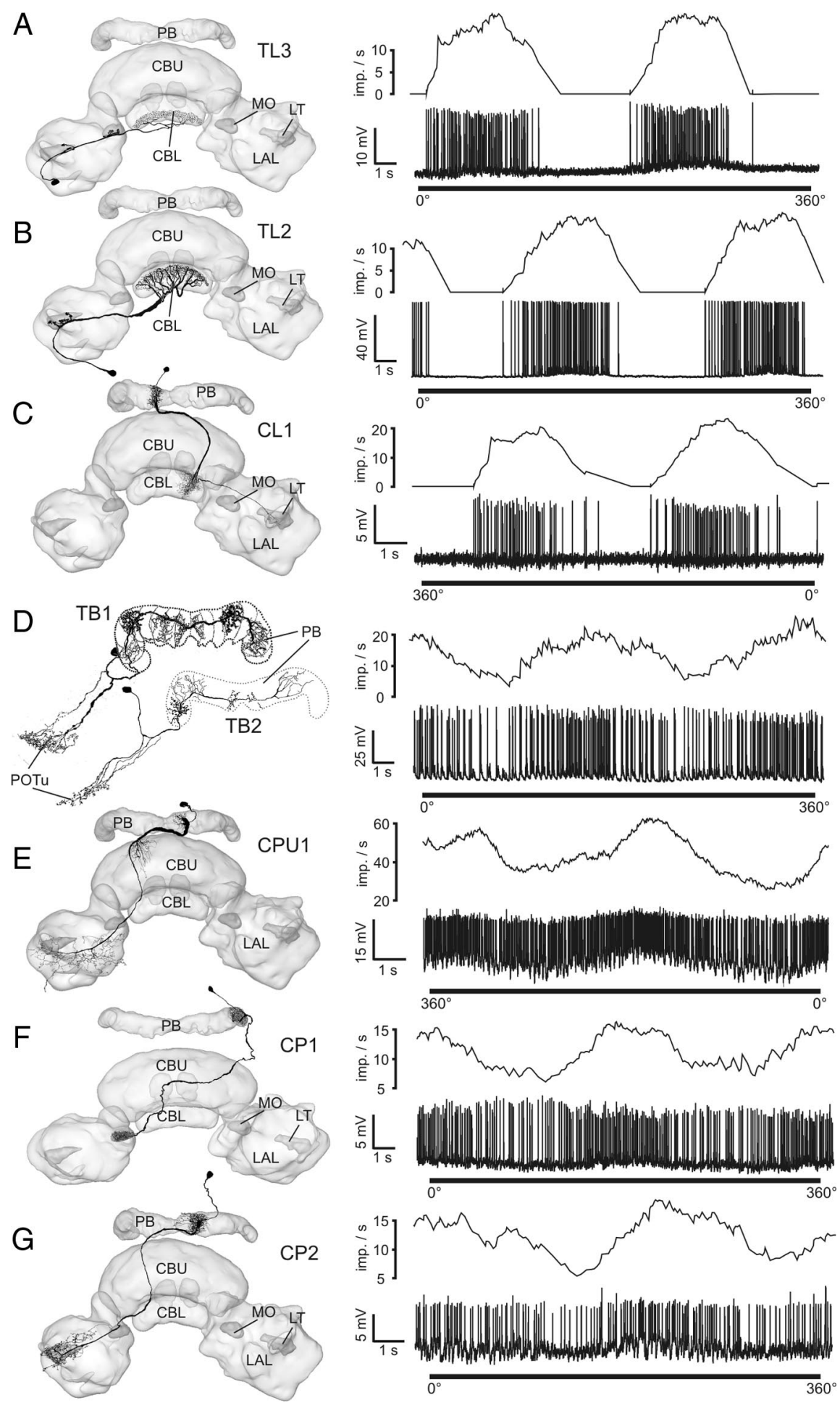

Figure 1. Morphologies of major types of POL-neuron of the locust central complex and their neuronal responses to a $360^{\circ}$ rotation of a dorsally presented polarizer. Frontal camera lucida reconstructions, projected onto three-dimensional reconstructions of the central complex, are shown on the left. Spike trains (lower lanes) and mean activities (upper lanes; gliding average, bin size: $1 \mathrm{~s}$ ) are shown on the right. Morphological and physiological data belong to neurons from the same cell type. $\boldsymbol{A}$, TL3 neuron. $\boldsymbol{B}$, TL2 neuron. C, CL1a neuron. D, TB1/2 neurons, physiology from a TB1 neuron. $E$, CPU1 neuron. $F$, CP1 neuron. G, CP2 neuron. LT, Lateral triangle; M0, median olive; POTu, posterior optic tubercle. Neuron morphologies are modified from Träger et al. (2008) $(\boldsymbol{A}, \boldsymbol{B})$, Heinze and Homberg (2008) (C, E-G), Heinze and Homberg (2007) (D, top), and Heinze and Homberg (2009) (D, bottom).

procedure ensured that the neurons with the best signal-to-noise ratio, i.e., the most reliable tuning curve shape, were analyzed. Spike frequencies were calculated in $5^{\circ}$ bins, and polarized-light responses were normalized to the peak activity within each $180^{\circ}$ rotation. After aligning all responses within a window of $180^{\circ}$ (minimal activity at $0^{\circ}$, maximal activity at $\left.90^{\circ}\right)$, the mean of each bin was calculated. This procedure was repeated for all five neurons and the average value $( \pm \mathrm{SD})$ of the considered cell type was determined for each $5^{\circ}$ bin.

Evaluation of receptive fields. For stimuli presented at different elevation, the response amplitudes were calculated. For each neuron, these values were normalized with respect to the largest value, i.e., the center of the receptive field, and thereafter plotted against the elevation. Because different elevations were tested in different recordings, average receptive fields could not be calculated directly. After assessing the raw data, we concluded that the receptive fields of all neuron types did not show centersurround organization or multiple peaks (with the possible exception of TL3 neurons), but response amplitudes rose and fell smoothly from background variability to maximal response amplitude in the receptive field center. This legitimized the interpolation of missing data for individual receptive fields, and eventually the calculation of mean response amplitudes at all elevations.

\section{Results}

\section{Central-complex POL-neurons}

The results are based on 116 intracellular recordings combined with dye injection for identification of the neuron type (supplemental Table 1, available at www. jneurosci.org as supplemental material). The recordings could be assigned to 12 different types of CC neurons, all of which have been shown to respond to dorsally presented polarized light before (Fig. 1) (Vitzthum et al., 2002; Heinze and Homberg, 2007, 2009). In response to a rotating polarizer placed in the zenith above the animal, neurons modulated their firing rate in a sinusoidal manner (Fig. 1). Each neuron preferred a particular $E$-vector orientation, corresponding to maximal spiking activity $\left(\Phi_{\max }\right.$ value) (supplemental Table 1, available at www. jneurosci.org as supplemental material).

Based on morphological evidence and the distribution of $\Phi_{\max }$ values, we proposed a particular scheme of connectivities within the CC polarization vision network (Heinze and Homberg, 2009). Here, we examined the physiological response characteristics of all major cell types of this network and provide independent evidence for different roles of these cells in processing polarized light information in the CC.

Background firing properties, response amplitudes and firing variability

First we compared the quantified responses of the CC POLneurons to a dorsally rotating polarizer. The resulting $R$ value measures the summed deviation from mean activity during each 

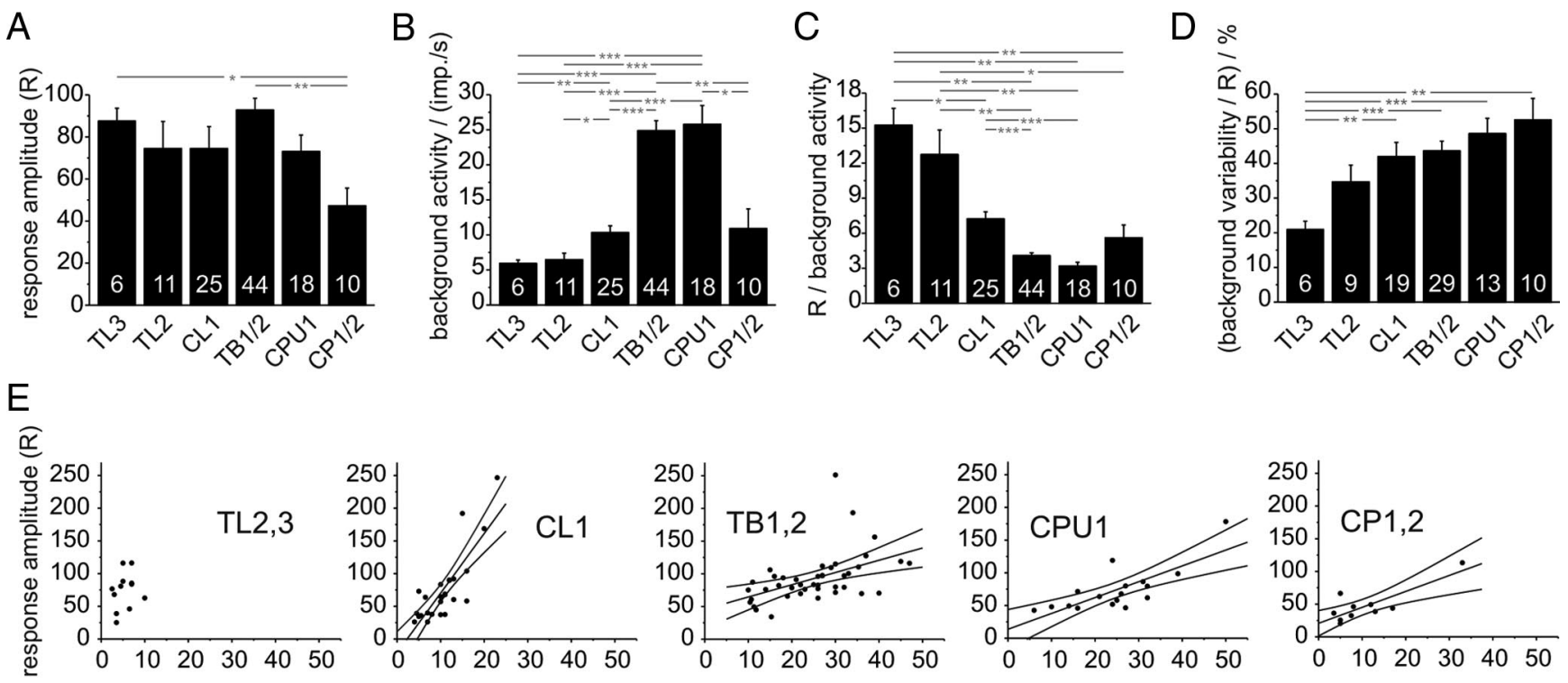

background activity / (impulses/s)

Figure 2. Response amplitudes and background activities in the different types of POL-neuron. $A$, Mean response amplitudes of all major types of neuron. $\boldsymbol{B}$, Background firing rates for each group of neuron. C, Ratios of response amplitude and background firing rate for each group of neuron. This ratio provides an estimate of the information content of the frequency modulations during a rotation of the polarizer. $\boldsymbol{D}$, Variability in background spiking activity. Means of $R$ values of background activity, normalized to the absolute response amplitude of each neuron, are plotted. The resulting values indicate how much variability during stimulation is due to spontaneous variability. Asterisks indicate significant differences as revealed by ANOVA analysis with Games-Howell post hoc test. Significance levels: ${ }^{*} p<0.05 ;{ }^{* *} p<0.01 ;{ }^{* * *} p<0.001$. Numbers of recordings are indicated in each bar. Error bars represent SE. E, Relation between response amplitude and background activity. The graphs show all recordings grouped according to main cell type. Linear regression lines (with $95 \%$ confidence intervals) are presented for each group of neurons. There was no statistical difference between the linear regressions for TB, CPU1, and (P neurons (test for equality of slopes, using analysis of covariance; $p=0.767$ ). TL neurons did not show significant linear correlation, while the regression line for CL1 neurons was distinct from all remaining regressions ( $p<0.001$ for all three cell types). Data for linear regressions: $\mathrm{TL2} / 3, p=0.47, R_{\text {cor }}=0.23 ; \mathrm{CL1}, p<0.0001$, $R_{\text {cor }}=0.82 ; \mathrm{TB} 1,2, p=0.0014, R_{\text {cor }}=0.48 ; \mathrm{CPU} 1, p=0.00036, R_{\text {cor }}=0.76 ; \mathrm{CP} 1 / 2, p=0.004, R_{\text {cor }}=0.82$.

filter rotation. The $R$ value increases with increasing frequency modulation during a polarized-light response, but contains no information about the directedness of this response. Therefore, this analysis was independent from the test for significant deviation from randomness by means of circular statistics (Rayleightest for axial data). Mean $R$ values were calculated for each neuron and the distribution of these values was compared between neuron types (supplemental Fig. 1, available at www. jneurosci.org as supplemental material). Based on morphological similarity and lack of significant differences in $R$ values and background activity (ANOVA, data not shown) we combined data on the subtypes of CL1 neurons, CPU1 neurons, TB neurons, and CP neurons (Fig. 2). TL2 and TL3 neurons were not combined, because they showed distinct reactions to more complex stimuli considered later. The mean $R$ values of all cell types were not statistically different from each other (ANOVA) (Fig. 2A), except for CP neurons, which showed significantly lower response amplitudes than TL3 and TB neurons.

In contrast to $R$ values, background activities differed significantly between several groups of POL-neurons (ANOVA) (Fig. $2 B)$. Lowest background firing rates were present in TL neurons (TL3: $5.9 \pm 0.5 \mathrm{impulses} / \mathrm{s}$, mean \pm SE; TL2: $6.5 \pm 0.9$ impulses/ s), highest rates were observed in TB neurons and CPU1 cells (TB: $24.9 \pm 1.4 \mathrm{impulses} / \mathrm{s}$; CPU1: $25.8 \pm 2.7 \mathrm{impulses} / \mathrm{s})$, and intermediate firing rates occurred in CL1 neurons (10.3 \pm 1.0 impulses/s) and CP neurons $(10.9 \pm 2.8$ impulses/s).

As frequency modulation during polarized-light responses is always superimposed upon background activity, the same response amplitude occurring at low or high background firing rates can be expected to have different effects on postsynaptic neurons. Therefore, we next analyzed the ratio of response am- plitude versus firing rate. When plotting these parameters for all neurons analyzed, each group of cells covered a certain region within this two dimensional parameter space (Fig. 2E). Analysis of each group of neurons showed, again with the exception of TL neurons, that the response amplitude and the background firing rate are linearly correlated, meaning that increased background activity was mirrored by higher $R$ values (Fig. $2 E$ ). Interestingly, the slope of the regression line depended partly on the type of neuron considered. Very similar values were obtained for TB, CPU1, and CP neurons (no significant difference in slope with ANCOVA, $p=0.767$ ), but CL1 cells showed a much faster increase in response amplitude with increasing background activity ( $p<0.001$ in all cases), placing them between TL neurons and the remaining cell types. Statistically comparing the ratios of $R$ values to background activity (ANOVA) confirmed this observation, showing that both types of TL neuron, as well as CL1 cells, had significantly higher ratios than TB and CPU1 cells (Fig. 2C). TL3 cells were also significantly different from CL1 cells, whereas both TL cells differed from CP neurons.

As the examined POL-neurons occur at different stages of information processing within the CC network, we analyzed the variability of polarized light responses in the different groups of neurons. To obtain a measure of intraneuronal variability of responses, $R$ values were obtained for each individual rotation of the polarizer. The SD of these values was calculated and normalized against the mean $R$ value of that cell. The resulting values indicate the relative variability in response amplitude of each neuron. Comparison of these values between the cell types did not reveal significant differences (supplemental Fig. 2, available at www.jneurosci.org as supplemental material) and ranged between 10 and $20 \%$ of the absolute response amplitude. 

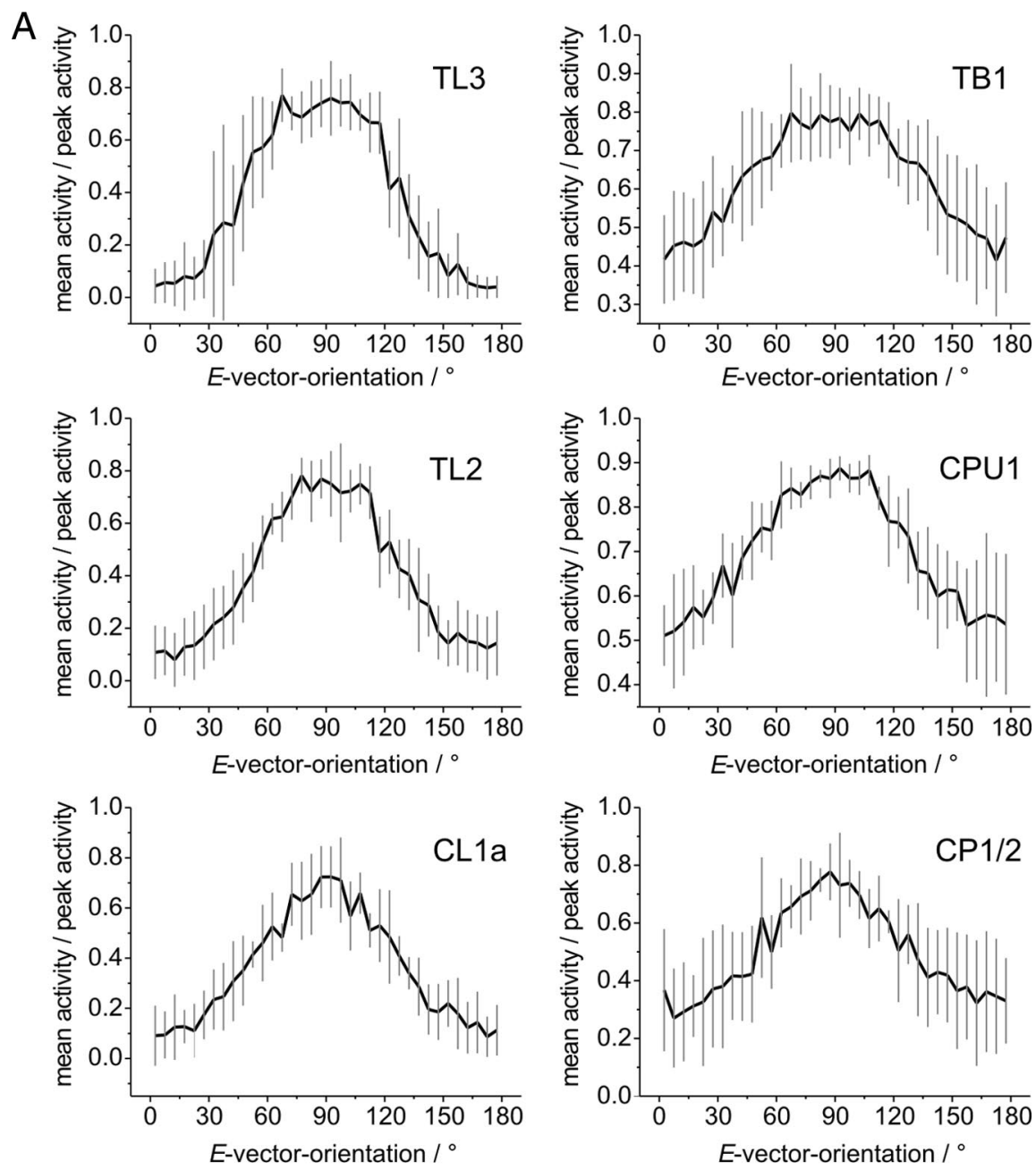

B

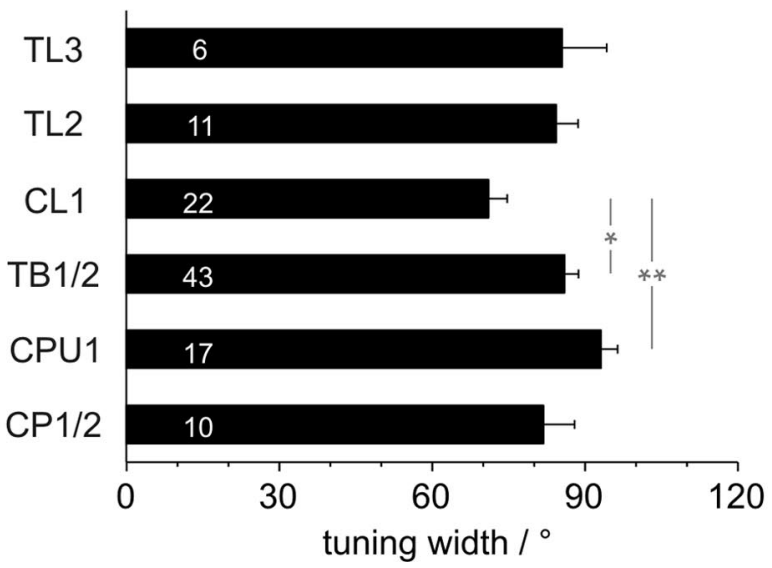

Figure 3. Characteristics of tuning curves of different types of POL-neuron. $A$, Average shapes of tuning curves (means \pm SD, bin size: $5^{\circ}$ ) from five neurons with the largest response amplitude in each category (supplemental Fig. 3, available at www. jneurosci.org as supplemental material). Tuning curves were normalized to peak activity during each rotation, manually aligned, averaged within each recording, and thereafter averaged across neurons. Plots are scaled from minimal to maximal activity. $\boldsymbol{B}$, Comparison of mean tuning widths at half maximal activation. Numbers of recordings are indicated in each bar. The tuning widths of some recorded neurons could not be determined reliably, because of low response amplitudes and ambiguous values of half maximal activation. Therefore the numbers are lower than in Figures $2 A-D$. Asterisks indicate significant differences revealed by ANOVA analysis combined with Tukey-HSD post hoc test. Significance levels: ${ }^{*} p<0.05 ;{ }^{* *} p<0.01$. Error bars represent SE.

Finally, we analyzed the magnitude of background variability in the different groups of neuron. We reasoned that the information content of a polarized-light response depends on the magnitude of frequency fluctuations occurring without the presence of the stimulus. To quantify background variability, we calculated the $R$ value of a spike train during periods when no stimulus was applied, and normalized these values for the response amplitude of that neuron (Fig. 2C). The results showed a general tendency of increasing background variability from lowest values in TL3 and TL2 neurons, to intermediate values in CL1 and TB neurons, and highest values in CPU1 and $\mathrm{CP}$ neurons. ANOVA revealed significantly lower background variability in TL3 cells, compared with all other cell types except TL2 cells. This implies that in CP neurons $>50 \%$ of the frequency modulations observed during polarized-light stimulations are due to background fluctuations in spiking activity, as opposed to only $21 \%$ in TL3 neurons.

Together, these analyses promote the separation of POL-neurons into three major groups: TL cells, CL1 cells, and TB/ CPU1 neurons. TL2 and TL3 neurons shared low background activity, high relative response amplitudes and the best signal-to-noise ratio. At the other end, high background activities, low relative response amplitudes, and lowest signalto-noise ratios were found in $\mathrm{TB}$ and CPU1 cells. Intermediate values were observed in CL1 neurons. With some uncertainty, CP neurons may be grouped together with $\mathrm{TB}$ and CPU1 cells.

\section{Tuning curves}

To further examine differences in the considered groups of POL-neurons, the shape and width of tuning curves were analyzed. The response curves of the five neurons with the highest response amplitude were selected for each cell type (supplemental Fig. 3, available at www.jneurosci.org as supplemental material), binned in $5^{\circ}$ bins, normalized to peak activity, and aligned with respect to their $\Phi_{\max }$ values (Fig. $3 A$ ). Several distinct features were observed when comparing the different types of POL-neuron. Despite their similar morphology and firing characteristics, TL2 and TL3 neurons differed in the shape of their tuning curves. TL3 cells showed a very steep rise of activity between maximal and minimal activation, resulting in an almost rectangular, all-or-nothing like response curve, with a plateau of peak activity covering an angle of $\sim 60^{\circ}$. The rising slope in activity was much gentler in TL2 cells, resulting in a more sinusoidal shape with a narrower plateau of peak activity. A distinctly triangular shape was found in CL1a cells. These neurons showed the narrowest region of peak activity, providing the most precise $E$-vector coding of all considered cell types. TB1 and CPU1 neurons were modulated only in the upper half of their 
activity range and exhibited a broad plateau-like tuning combined with a gentle rise and fall between $\Phi_{\text {max }}$ and $\Phi_{\text {min }}$ values. The overall shape of the tuning curve was slightly asymmetrical in about half of the included CPU1 neurons. Here, either the rising or the falling slope of the curve was convex and graded, whereas the opposite slope was more abrupt and concave. Finally, CP1/2 neurons were most variable and possessed the least distinct tuning curves with an overall sinusoidal appearance.

These qualitative observations were supported by measurements of the tuning width at half-maximal activation (Fig. $3 B$ ). On average, largest values were found in CPU1 neurons $\left(93^{\circ} \pm 3.3^{\circ}\right)$, whereas the narrowest tuning was present in CL1 cells $\left(71^{\circ} \pm 3.7^{\circ}\right.$; significantly smaller with ANOVA analysis). The remaining types of neuron had a similar tuning width between $82^{\circ}$ and $86^{\circ}$, and only TB1/2 cells had a significantly broader tuning than CL1 neurons (Fig. 3B). Therefore, for most neurons, regions of inhibition and excitation are nearly equally distributed within the $180^{\circ} \mathrm{E}$-vector range. Only CL1 neurons exhibit consistently more inhibition, resulting in an increased coding precision within the range of activation. Together, the analysis of tuning curves established a distinct behavior of CL1 neurons and a clear difference between TL2 and TL3 cells. As in the previously described firing characteristics, tuning curves of TB1 and CPU1 neurons had very similar characteristics.

\section{Ocular dominance}

We next analyzed the ocular dominance of all groups of CC POLneurons to elucidate how much information each neuron receives from either eye. Data were obtained from 26 recordings with binocular and monocular stimulation.

Most neurons showed strong responses to a rotating polarizer at monocular and binocular stimulation without obvious differences in response characteristics. The only exceptions were TL3 neurons, which responded exclusively to stimulation through the ipsilateral eye. These observations were confirmed when analyzing the response amplitudes ( $R$ values) of each neuron during monocular and binocular stimulations. For each neuron, $R$ values for monocular stimulations were normalized to the binocular response amplitude and thereafter pooled within each group of neurons (Fig. 4A). Comparison of $R$ values for monocular and binocular stimulations (Mann-Whitney $U$ test, significance level: 0.05 ) revealed that all neurons except TL3 cells responded equally well to ipsilateral and contralateral stimuli. In three recordings from CPU1 neurons and in one recording from a CL1 cell, however, the responses to the rotating polarizer were not significantly different from randomness in one of the monocular stimulus presentations, possibly due to increased variability during prolonged recordings (data not shown).

After evaluating the response amplitudes, differences in $E$-vector tuning for monocular and binocular stimulations were
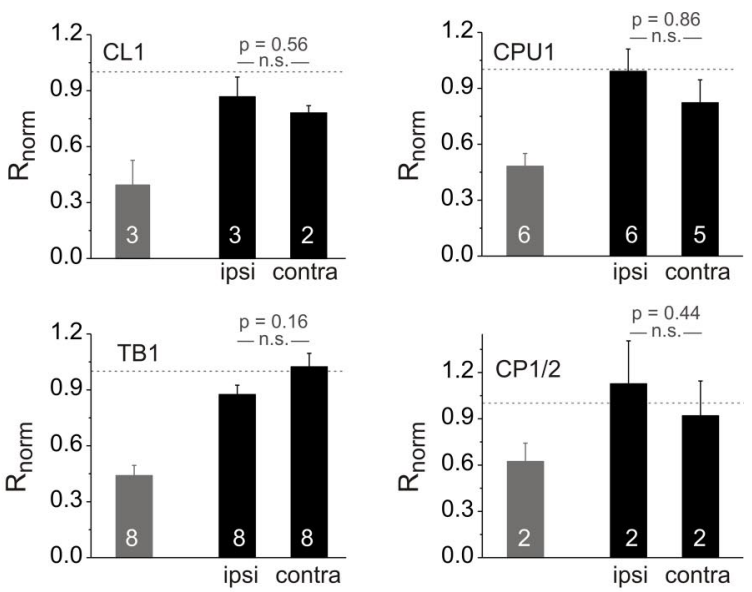

C
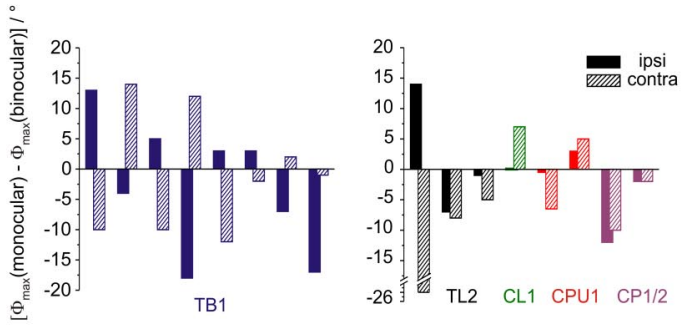

Figure 4. Ocular dominance of the different types of POL-neuron. $\boldsymbol{A}$, Response amplitudes for monocular stimulation with a

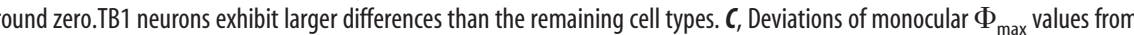
TB1 neurons are presented in the left graph, all remaining cell types in the right graph. In most TB1 neurons binocular values were between the monocular stimulations, whereas this occurred only once in all remaining cell types.

analyzed. Only stimulations leading to responses that were significantly different from randomness were included. As expected, $\Phi_{\max }$ values of TL3 neurons were nearly identical for ipsilateral and binocular stimulations (maximal deviation: $6^{\circ}$ ). This was in tune with the finding that these neurons received exclusively ipsilateral input. In 16 additional recordings, significant $\Phi_{\max }$ values were obtained for all three stimulus situations. These cells could be divided into two groups; the TB1 neurons and all remaining neurons. In TB1 cells, $\Phi_{\max }$ values for binocular stimulation were between the $\Phi_{\max }$ values for ipsilateral and contralateral stimulation (Fig. $4 C$; seven out of eight cells). For most of the remaining cells this was not the case, and both monocular $\Phi_{\max }$ values were either larger $(1 \times)$ or smaller $(5 \times)$ than the binocular value (Fig. $4 C$ ). In six recordings, only one monocular stimulus was tested $(2 \times \mathrm{CL} 1,4 \times \mathrm{CPU} 1)$. In these neurons, the binocular $\Phi_{\max }$ value was always larger than the monocular one (data not shown). The difference between TB1 neurons and the remaining cells was also reflected in the distribution of the differences between ipsilateral and contralateral $\Phi_{\max }$ values (Fig. $4 B)$. Here, TB1 neurons generally showed larger $\Delta \Phi_{\max }$ values than all other cell types. Interestingly, values were distributed symmetrically around zero, indicating that ipsilateral and contralateral $\Phi_{\max }$ values were larger in equal numbers of recordings. Quantitative analysis of the absolute $\Delta \Phi_{\max }$ values resulted in a mean value of $16.4^{\circ} \pm 2.7^{\circ}(\mathrm{SE})$ for TB1 cells and $7.6^{\circ} \pm 4.6^{\circ}$ for the remainders. The two distributions were significantly different from each other (Mann-Whitney $U$ test, $p=0.021$ ). To test 

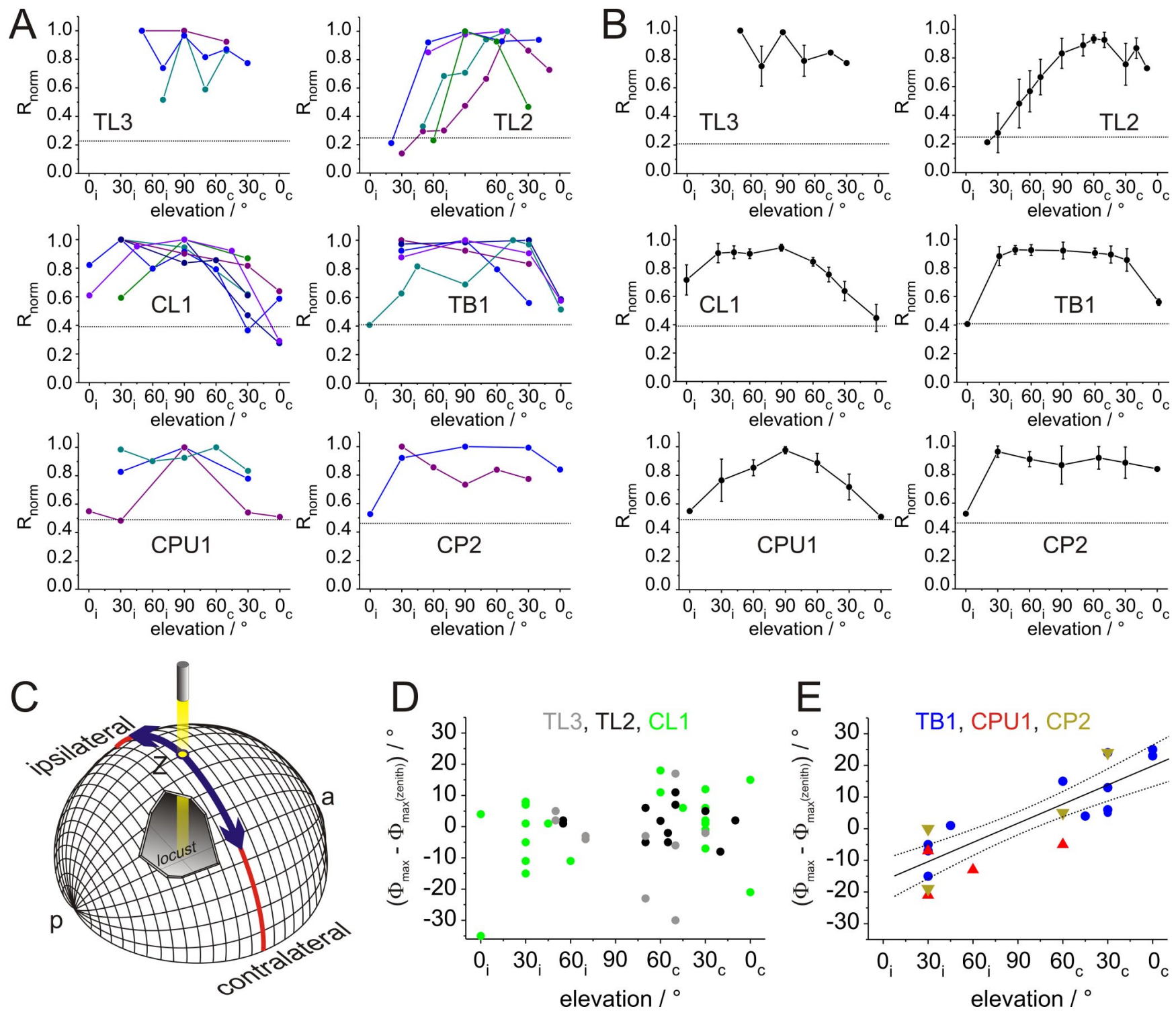

Figure 5. Receptive field properties of POL-neurons. $A$, Response amplitudes of the different types of POL-neuron plotted against elevation of polarized-light stimulus. Elevation is plotted with respect to the location of the soma and was sampled along the meridian orthogonal to the longitudinal body axis of the locust. Values were normalized to the largest $R$ value of each neuron (i.e., the receptive field center). Individual recordings are distinguished by different colors. Data points are connected by lines for better visibility. Dotted lines indicate background variability for each neuron type (from Fig. 4), which have been renormalized with respect to the mean receptive field center in each cell type. B, Average receptive fields for the examined groups of POL-neurons. Dotted lines, background variability. Values are means \pm SE. Missing values in individual recordings were interpolated linearly to allow for averaging at all elevations. C, Schematic representation of stimulus display. The locust is located in the center of the sphere, oriented along the anterior (a)-posterior (p) axis. Stimuli were moved along the meridian (blue) orthogonal to the body axis. Ipsi- and contralateral refer to neurons with cell body in the left hemisphere of the brain. $\boldsymbol{D}, \boldsymbol{E}$, Deviations of $\Phi_{\max }$ values at different elevations from zenithal $\Phi_{\max }$ values for TL3, TL2, and CL1 neurons (D), and for TB1, CPU1, and CP2 neurons ( $E$ ). Differences in $\Phi_{\max }$ values are plotted against elevation. A significant correlation was observed for the combined values of TB1, CPU1, and CP1 ( $R_{\text {cor }}=0.84$, $t$ test against the slope of $0, p<0.00001)$, indicated in $\boldsymbol{E}$ by the regression line with $95 \%$ confidence intervals. No correlation was observed for neurons in $\boldsymbol{D}\left(R_{\text {cor }}=0.19, p=0.23\right)$.

whether the responses to either ipsilateral or contralateral stimuli were more similar to the binocular responses, we also examined the distributions of the differences between $\Phi_{\max }$ values of both monocular responses from the binocular response (data not shown). Both resulting distributions were concentrated around zero, i.e., identical values for monocular and binocular stimulations, with a slight shift toward smaller monocular values (ipsilateral: $-2.4^{\circ} \pm 1.8^{\circ}$, Fig. $4 C$; contralateral: $-4.0^{\circ} \pm 2.6^{\circ}$ ). There was no significant difference between the two distributions (twosided Student's $t$ test, $p=0.61$ ). Because the mean $\Phi_{\max }$ values at ipsilateral and contralateral stimulation deviated equally from the $\Phi_{\max }$ values during binocular stimulation, input from neither eye dominated over the other eye across the population of recorded cells.

\section{Structure of receptive fields}

As most neurons received input from both eyes, we tested whether the different types of POL-neuron could be distinguished further by the structure of their receptive fields. Therefore, rotating $E$-vectors were presented at different elevations along the right-left meridian, covering a range from $0^{\circ}$ elevation on either side of the animal to the zenith $\left(90^{\circ}\right)$ (Fig. 5C). At least three different positions (always including dorsal presentation) were tested in each of 25 recordings, including all major types of POL-neuron $(3 \times \mathrm{TL} 3,5 \times \mathrm{TL} 2,7 \times \mathrm{CL} 1,5 \times \mathrm{TB} 1,3 \times \mathrm{CPU} 1$, $2 \times \mathrm{CP} 2)$. For estimating the lateral extension and shape of receptive fields, the response amplitude was calculated at each position and was normalized to the maximal value in each neuron. In all recorded cell types, receptive fields were large (Fig. $5 A$ ). The lat- 

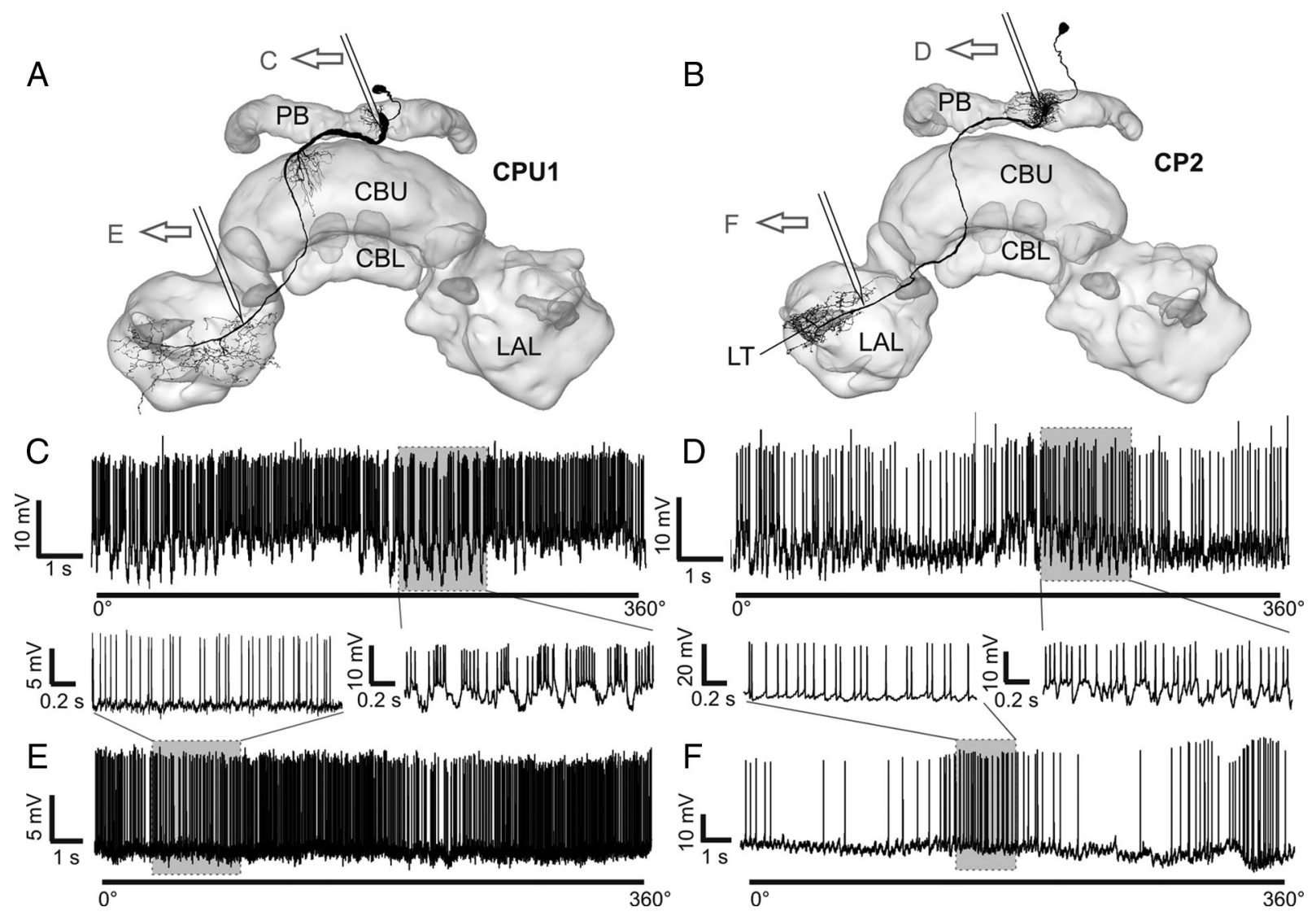

Figure 6. Indication of presynaptic and postsynaptic arborizations by recordings from different regions of $C P U 1$ and $(P 2$ neurons. $A, B$, Frontal reconstructions of a $(P U 1(A)$ and $C P 2$ neuron $(B)$, projected onto a three-dimensional reconstruction of the $C($. Approximate recording sites of the recording traces in $C-F$ are indicated. $C$, Recording trace from arborizations of a CPU1 neuron in the PB during zenithal rotation of the polarizer. Postsynaptic, graded potentials are visible (enlargement of shaded area is shown in the inset). $\boldsymbol{D}$, Recording trace from PB-arborizations of a CP2 neuron during a rotation of the polarizer. Postsynaptic potentials are clearly visible (enlarged in the inset). $\boldsymbol{E}, \boldsymbol{F}$, Recording traces obtained from the vicinity of the LAL from a $(P U 1$ neuron $(\boldsymbol{E})$ and a $C P 2$ neuron $(\boldsymbol{F})$. Enlargements emphasize the even baseline without major graded potential changes. LT, Lateral triangle. Neuron morphologies in $\boldsymbol{A}$ and $\boldsymbol{B}$ are modified from Heinze and Homberg (2008).

eral size ranged from $\sim 60^{\circ}$ in a TL2 neuron to $\sim 165^{\circ}$ in a CP2 cell. With the possible exception of TL3 neurons, no complex structure, such as center-surround organization, was apparent.

As raw data for each type of neuron appeared sufficiently similar, mean receptive fields were calculated for comparison of neuron types (Fig. 5B). TL2 and CL1 neurons had eccentric fields with peak activation either in the contralateral field of view (TL2) or in the ipsilateral field of view (CL1). TB1, CPU1, and CP2 neurons had zenith-centered receptive fields. For TL3 neurons, no definite statement can be made due to low number of recordings and high variability. The widths of the receptive fields were calculated as the angles resulting in half-maximal response amplitude in relation to variability in background spiking activity. Thus, background variability rates (Fig. $2 B$ ) had to be renormalized to maximum response amplitude of the receptive field center. This calculation revealed very similar background variability for TL2 and TL3 neurons, and indicated that the response strength of TL2 cells was underestimated when only considering dorsal stimulations. When comparing mean lateral extension of receptive fields, the smallest sizes were found in TL2 $\left(100^{\circ}\right)$, and very large receptive fields in CL1, TB1, CPU1, and CP2 neurons $\left(135^{\circ}, 150^{\circ}, 120^{\circ}\right.$, and $\left.150^{\circ}\right)$. TL3 cells covered at least $90^{\circ}$ along the tested meridian.

Surprisingly, significant activation was in some cases observed even at $0^{\circ}$ elevation. This was most prominently found in the ipsilateral field of view of CL1 neurons. This indicates that the neurons are sensitive to polarized light from the ze- nith down to the horizon, but since alignment of the animals with respect to the perimeter was done by eye judgment, minor deviations from $0^{\circ}$ (up to $5^{\circ}$ ) cannot be excluded for some of the experiments. Indications of subpopulations within cell types were observed in TL2 cells: two neurons responded almost exclusively to stimuli in the contralateral field of view; in two other cells the visual fields ranged $\sim 30^{\circ}$ across the midline, and the fifth cell responded in a narrow contralateral range close to the midline (Fig. 5A).

In addition to response amplitudes within the receptive fields, we also analyzed the distribution of $\Phi_{\max }$ values at different elevations (Fig. 5D,E; supplemental Tables 2-4, available at www.jneurosci.org as supplemental material). When calculating the absolute deviation of $\Phi_{\max }$ values in each tested position from the $\Phi_{\max }$ value in the zenith, all POL-neurons fell into one of two categories. The values for the first group (TL3, TL2, and CL1 neurons) were concentrated around zero (Fig. 5D). Variability increased toward lower elevations, as would be expected from the lower response amplitudes. Increasing deviations at lower elevations were also found in the second group. However, in TB1, CPU1, and CP2 neurons the deviation from zenithal $\Phi_{\max }$ values also depended on the hemispheric side of the stimulus (Fig. 5E). For stimulations in the ipsilateral field of view, $\Phi_{\max }$ values were consistently smaller than in the zenith, whereas they were consistently larger in the contralateral hemisphere $\left(R_{\mathrm{cor}}=\right.$ $0.84, t$ test against the slope of zero: $p<0.00001)$. No such hemi- 


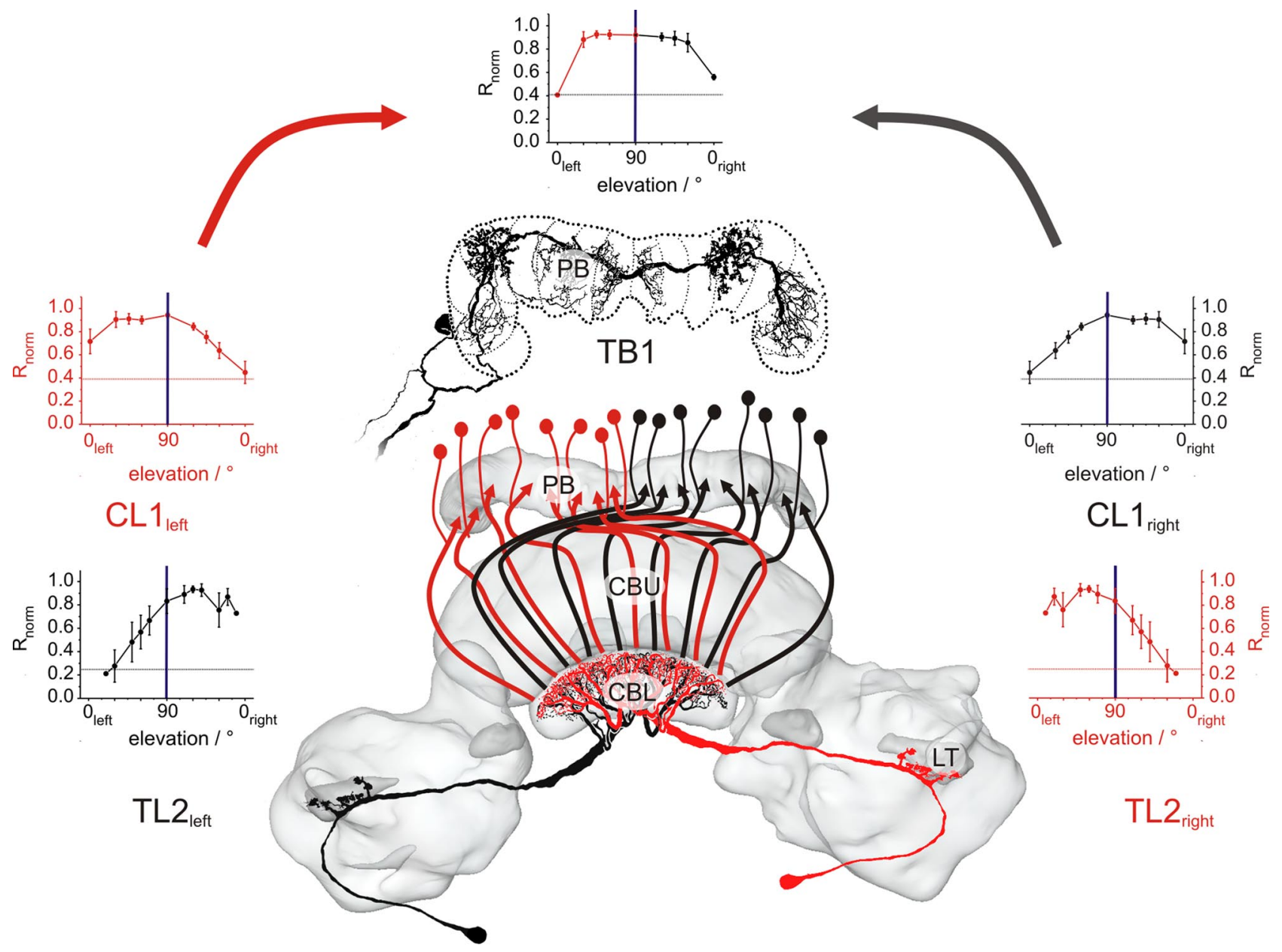

Figure 7. Model of neuronal wiring principles derived from receptive field data superimposed on a three-dimensional reconstruction of the central complex. Input neurons (TL2) have relatively small, contralaterally centered receptive fields, i.e., neurons in the left brain hemisphere have receptive fields centered in the right sky hemisphere (black) and vice versa for the right brain hemisphere (red). CL1 neurons have slightly larger, ipsilaterally centered receptive fields, implying midline crossing connections between TL2 and CL1 neurons (colors indicate the source of polarized light information). CL1 neurons are represented by arrows following the anatomical trajectories of CL1 cells. The smooth arborizations of TB1 neurons (top, projections to the posterior optic tubercle omitted) on either side of the PB-midline provide the potential targets for CL1 cells. Therefore, the bilaterally symmetrical receptive fields of TB1 neurons can be explained by integration of signals from at least two CL1 cells, one from each hemisphere. The morphology of the TB1 neuron is modified from Heinze and Homberg (2007).

sphere dependency was found in the first group (TL3, TL2, and CL1 neurons; $\left.R_{\text {cor }}=0.19, p=0.23\right)$.

\section{Physiological evidence for input and output regions}

To obtain information about the direction of information flow within the neurons, we compared recordings from different recording sites within the same cell types. As potential input and output areas are in close proximity in TB1/2 neurons, and also to some extend in TL2/3 neurons, this could be achieved only in columnar cells. Neurons were recorded either from the PB or from the vicinity of the lateral accessory lobe (LAL) (Fig. 6). Very large postsynaptic potentials (PSPs) were observed consistently in CPU1 neurons ( $n=15$; all subtypes), when they were recorded from the PB (Fig. 6C), whereas no PSPs were present in recordings from their axons in the LAL $(n=3)$. Similarly, CP2 neurons showed strong PSPs when recorded from the $\mathrm{PB}(n=3)$ but none when recorded from the LAL $(n=1)$ (Fig. $6 D)$. CP1 cells could not be recorded from the $\mathrm{PB}$, but none of the recordings obtained from the LAL showed PSPs. For CL1 neurons, the difference was not as distinct. In only one recording of a CL1b/d neuron from the PB, strong PSPs were observed. In most other recordings from CL1 cells, no or small PSPs were present independently of the recording site.

\section{Discussion}

At least 13 different types of neuron of the locust CC are sensitive to polarized light. While all of these cells showed polarization opponency, cell-type specific differences were found in background activity, variability in spiking, signal-to-noise ratio, tuning precision, ocular dominance, and receptive field structure. Together with data on neuronal morphologies, the distribution of smooth and varicose terminals, and physiological support for the association of dendritic input regions with smooth ramifications, a picture of network connectivity and information flow within the polarization vision system of the CC emerges.

\section{Information flow through the $\mathrm{CC}$}

Anatomical position and physiological characteristics indicate that tangential neurons of the CBL (TL2, TL3) are at the input stage of the CC, whereas CPU1, TB1/2, and CP1/2 neurons are near the output. CL1 neurons show intermediate characteristics and likely link input to output elements. Neurons at the input stage (TL2, TL3) showed highest relative response amplitudes while their absolute responses ranged from total inhibition to the maximally occurring frequencies. This suggests that they are specialized for $E$-vector coding. As these neurons show very low background activity and variability, virtually all activity origi- 
nates from polarization input. Neurons with similar characteristics are also present in crickets (Sakura et al., 2008) and support a common design of the input pathway to the polarization-vision network in the CC of orthopteroid insects.

Between early stage and late stage POLneurons, background variability increased while response variability remained constant. Therefore, sources of background activity not controlled by the polarizationvision network are likely. CPU1 neurons probably receive polarization-independent input through their $\mathrm{CBU}$ ramifications (Heinze and Homberg, 2008). This is supported by the finding that $\mathrm{CBU}$ tangential cells in Drosophila are required for memory of visual object parameters and, thus, code sensory information different from polarized light (Liu et al., 2006). As rotating $E$-vectors modulate TB1/2 and CPU1 cells only in the upper half of their activity range, i.e., they are not totally silenced during $\Phi_{\min }$, these stimuli alone are insufficient to drive these neurons optimally. In more natural stimulus situations these cells might be converging sites for multisensory information and mediate the association of head orientation with information about behaviorally relevant sensory features.

Ocular dominance analysis revealed that neurons of the anterior optic tubercle receive polarized light input predominantly through the ipsilateral eye (Pfeiffer et al., 2005). POL-neurons of the CC that respond to stimuli from both eyes, therefore, constitute later processing stages. This is in tune with the situation in the mammalian visual system, where binocularity emerges for the first time in the primary visual cortex, several synapses away from the sensory periphery (Horton, 2006).

The transition of receptive fields from medium-sized and eccentric to large and zenith-centered, likewise, supports a direction of information flow from TL2 and CL1 neurons to TB1, CPU1 and $\mathrm{CP} 2$ neurons. The receptive fields in the latter neurons cover the complete width of the sky, implying that their responses to polarized light are independent of stimulus position. This agrees well with the concept of position invariance at higher levels of the mammalian visual cortex. Here, receptive field size increases with increasing distance from the sensory periphery, until neurons finally respond to specific aspects of the environment, independently of stimulus position (Rolls, 2007).

\section{Two parallel input pathways to the CC}

The differences in response characteristics between TL2 and TL3 neurons suggest that two separate input pathways link the LAL with the CBL. While the monocular TL3 neurons transfer information from the median olive and lateral triangle to layers $4-5$ of the CBL, all TL2 neurons studied here receive their binocular input in the lateral triangle and project to layer 2 of the CBL. CL1a neurons have fine arborizations in layer 2 (Heinze and Homberg, 2008) and may, therefore, be the postsynaptic partners of TL2 neurons. Because neurons with smooth arborizations in CBL- layers served by TL3 cells are not known to date, parts of the varicose endings of CL1 neurons in these layers might in fact be postsynaptic. If so, both pathways would converge at the level of CL1 cells.

\section{CL1 neurons-key players of the network}

Despite their opposite polarity, CL1a and CL1b/c/d neurons could not be distinguished physiologically. This suggests that all CL1 cells code information at the same computational level and constitute a set of cells allowing for bidirectional information exchange between the CBL and the PB. They signal E-vector information from one sky hemisphere with the greatest precision of all cell types and transfer it to a position-invariant population of head-orientation cells in the PB. Because CL1 neurons showed the sharpest $E$-vector tuning of all cells examined, they are promising candidates for "compass neurons," postulated by Sakura et al. (2008). Sakura et al. (2008) proposed the necessity of a gain control circuit in the polarization-vision network to insure constant response amplitudes at varying degrees of polarization. Gain control could, likewise, assess the importance of polarization vision relative to other sensory input depending on behavioral and motivational context. The dependency of response amplitudes on background activity might serve as the neuronal substrate for a gain control. Of all POL-neurons, CL1 neurons showed the steepest rise in response amplitude with increasing background activity. Neurons modulating their background activity would, therefore, dramatically change the influence of CL1 cells onto their postsynaptic partners in the PB and might also 
account for the switch between the polarization-sensitive and insensitive states of conditional POL-neurons (Heinze and Homberg, 2009). Polarization-insensitive TL5 neurons of the CBL (Vitzthum et al., 2002), which are probably dopaminergic (Wendt and Homberg, 1992), are promising candidates for this task.

\section{Transformation of visual fields}

At the input stage of the CC (TL2 neurons), receptive fields are centered in the contralateral hemisphere and have a size of $\sim 60^{\circ}$. This corresponds well to data for POL1 neurons in the cricket optic lobe (Labhart et al., 2001). As CL1 neurons are most likely postsynaptic to TL2 cells, the ipsilateral position of their receptive field centers suggests that TL2 neurons exclusively synapse onto contralateral CL1 neurons (Fig. 7). Because receptive fields were larger in CL1 cells than in TL2 cells, convergence of several TL2 neurons onto individual CL1 cells is likely. If CL1 neurons are presynaptic to late stage POL-neurons, at least one CL1 cell from each hemisphere must be sampled by a postsynaptic neuron to account for the transformation of ipsilateral receptive fields to bilaterally symmetric receptive fields. TB cells have smooth input processes in both hemispheres of the $\mathrm{PB}$ and are, therefore, ideally suited to achieve this integration (Fig. 7) (Heinze and Homberg, 2007). Supporting evidence for multiple spike initiation zones in TB cells comes from the regular observation of action potentials with multiple sizes (Fig. $1 D$ ). Once bilateral symmetry of receptive fields is reached in TB cells, this character is transferred onto CPU1 and CP cells, which share all major receptive field characteristics with $\mathrm{TB}$ neurons.

\section{Evidence for a $360^{\circ}$ azimuth map in the PB}

Zenithal $E$-vectors are represented topographically in the columns of the PB (TB1, CPU1, and CP1/2 cells; Heinze and Homberg, 2007) and cover a solar azimuthal range of $180^{\circ}$ across each PB hemisphere (Fig. 8). As E-vector orientation only indicates the solar/antisolar meridian, it is ambiguous with regard to the location of the solar versus antisolar sky hemisphere. Therefore it remained unclear whether the reported $E$-vector map covers a double representation of $180^{\circ}$ of azimuthal space, or is a true $360^{\circ}$ representation of azimuth. To resolve the $E$-vector ambiguity, neurons of the anterior optic tubercle of the locust integrate spectral cues with polarization information (Pfeiffer and Homberg, 2007). Whether and how this information is transferred to the CC remains to be shown. The distribution of $\Phi_{\max }$ values across the receptive field offers an additional possibility to solve this problem. In TB1, CPU1, and CP2 neurons, stimuli presented in the ipsilateral field of view elicited smaller $\Phi_{\text {max }}$ values than stimuli from contralateral directions. Consequently, neurons in the right brain hemisphere prefer stimulus situations in which $E$-vector angles in the right half of the sky are smaller than in the left half. As $E$-vectors in the sky are oriented along concentric circles around the sun, this matches the natural sky for solar elevations larger than $0^{\circ}$, when the locust faces an antisolar direction (Fig. 8, right). Likewise, E-vector tuning of neurons in the left brain hemisphere is matched when the locust faces the solar sky hemisphere (Fig. 8, left). Under the natural sky, peak activities in columnar neurons of the right $\mathrm{PB}$ hemisphere would, therefore, occur when the sun is behind the animal, whereas neurons in the left $\mathrm{PB}$ hemisphere would respond stronger when facing the sun, even though zenithal $E$-vectors are identical in both situations (Fig. 8). Because such tuning differences were not found in CL1 and TL neurons, position-invariant E-vector tuning in these cells is apparently transformed into head orientation tuning in later-stage neurons. Thus, for solar elevations different from $0^{\circ}$ (horizon), polarotopy in the $\mathrm{PB}$ is biased toward a $360^{\circ}$ azimuthal range around the animal, as suggested by Strauss (2002b).

\section{References}

Clements AN, May TE (1974) Studies on locust neuromuscular physiology in relation to glutamic acid. J Exp Biol 60:673-705.

Heinze S, Homberg U (2007) Maplike representation of celestial E-vector orientations in the brain of an insect. Science 315:995-997.

Heinze S, Homberg U (2008) Neuroarchitecture of the central complex of the desert locust: Intrinsic and columnar neurons. J Comp Neurol 511:454-478.

Heinze S, Homberg U (2009) Linking the input to the output: new sets of neurons complement the polarization vision network of the locust central complex. J Neurosci 29:4911-4921.

Homberg U (1991) Neuroarchitecture of the central complex in the brain of the locust Schistocerca gregaria and S. americana as revealed by serotonin immunocytochemistry. J Comp Neurol 303:245-254.

Homberg U (2004) In search of the sky compass in the insect brain. Naturwissenschaften 91:199-208.

Horton JC (2006) Ocular integration in the human visual cortex. Can J Ophthal 41:584-593.

Labhart T (1996) How polarization-sensitive interneurones of crickets perform at low degrees of polarization. J Exp Biol 199:1467-1475.

Labhart T, Petzold J, Helbling H (2001) Spatial integration in polarizationsensitive interneurones of crickets: a survey of evidence, mechanisms and benefits. J Exp Biol 204:2423-2430.

Liu G, Seiler H, Wen A, Zars T, Ito K, Wolf R, Heisenberg M, Liu L (2006) Distinct memory traces for two visual features in the Drosophila brain. Nature 439:551-556.

Müller M, Homberg U, Kühn A (1997) Neuroarchitecture of the lower division of the central body in the brain of the locust (Schistocerca gregaria). Cell Tissue Res 288:159-176.

Neuser K, Triphan T, Mronz M, Poeck B, Strauss R (2008) Analysis of a spatial orientation memory in Drosophila. Nature 453:1244-1247.

Petzold J (2001) Polarisationsempfindliche Neuronen im Sehsystem der Feldgrille Gryllus compestris: Elektrophysiologie, Anatomie und Modellrechnungen. PhD thesis, University of Zurich.

Pfeiffer K (2006) Coding of sky-compass information in neurons of the anterior optic tubercle of the desert locust Schistocerca gregaria. $\mathrm{PhD}$ thesis, Philipps-University Marburg.

Pfeiffer K, Homberg U (2007) Coding of azimuthal directions via timecompensated combination of celestial compass cues. Curr Biol 17: 960-965.

Pfeiffer K, Kinoshita M, Homberg U (2005) Polarization-sensitive and light-sensitive neurons in two parallel pathways passing through the anterior optic tubercle in the locust brain. J Neurophysiol 94:3903-3915.

Rolls ET (2007) The representation of information about faces in the temporal and frontal lobes. Neuropsychologia 45:124-143.

Sakura M, Lambrinos D, Labhart T (2008) Polarized skylight navigation in insects: model and electrophysiology of e-vector coding by neurons in the central complex. J Neurophysiol 99:667-682.

Strausfeld NJ (1999) A brain region in insects that supervises walking. Prog Brain Res 123:273-284.

Strauss R (2002a) The central complex and the genetic dissection of locomotor behaviour. Curr Opin Neurobiol 12:633-638.

Strauss R (2002b) Die übergeordnete Steuerung des Laufverhaltens durch das Insektengehirn, studiert mit Methoden der Drosophila-Neurogenetik. Habilitationsschrift, University of Würzburg.

Träger U, Wagner R, Bausenwein B, Homberg U (2008) A novel type of microglomerular synaptic complex in the polarization vision pathway of the locust brain. J Comp Neurol 506:288-300.

Vitzthum H, Müller M, Homberg U (2002) Neurons of the central complex of the locust Schistocerca gregaria are sensitive to polarized light. J Neurosci 22:1114-1125.

Wendt B, Homberg U (1992) Immunocytochemistry of dopamine in the brain of the locust Schistocerca gregaria. J Comp Neurol 321:387-403.

Williams JLD (1975) Anatomical studies of the insect central nervous system: a ground-plan of the midbrain and an introduction to the central complex in the locust, Schistocerca gregaria (Orthoptera). J Zool (Lond) 176:67-86. 